\title{
Working Memory and Functional Neuroimaging in Patients with Depression
}

\author{
Chaoqun Chen, Jian Chen, Liangtie Dai \\ Jinan university, Guangzhou, China \\ Email: dzlccq2012@163.com, chenjian@163.com, \\ dailiangtie@163.com
}

Received 10 August 2015; accepted 2 September 2015; published 7 September 2015

Copyright (C) 2015 by authors and Scientific Research Publishing Inc.

This work is licensed under the Creative Commons Attribution International License (CC BY). http://creativecommons.org/licenses/by/4.0/

(c) $\underset{\mathrm{EY}}{\mathrm{B}}$ Open Access

\begin{abstract}
Numerous studies have demonstrated that patients suffering from depression present impairment in working memory. This paper firstly introduced the concept of working memory, and then elucidated the deficits of working memory of patients with depression. It also reviewed the neuroimaging studies of working memory in patients with depression. In the final part, we further summarized the future research orientations. In short, exploration of working memory on the regulative mechanisms of depression and figuring out that working memory impairment is the cause or the result of depressive symptoms will be the core problem that the future research needs to address.
\end{abstract}

\section{Keywords}

Depression, Working Memory, Neuroimaging

\section{Introduction}

Depression is a kind of severe mental disorder which is of high incidence, high recurrence rate, high morbidity and high suicide rate. In recent years, an increasing number of studies have found that patients with depression would have different degrees of cognitive dysfunction in both stage of attack and remission. Most patients mainly show a decrease of learning ability, lack of efficiency, memory and attention impairments (Marazziti et al., 2010). Some scholars have suggested that working memory is the core of cognitive function. The related research has attracted more and more attention. This is an overview of status of research about working memory in patients with depression. 


\section{Working Memory in Patients with Depression}

\subsection{The Concept of Working Memory}

Working memory is an important part of cognitive function; it's the foundation of the learning and memory. Besides, it has a close relation with other cognitive behavior. The concept model of working memory was initially proposed by Baddeley and Hitch in 1974, it refers to a system of limited capacity which stores and processes information temporarily when people complete complex cognitive activities such as speech understanding, learning and reasoning. And working memory consists of three components: a central executive system and two buffers, namely "phonological loop" and "visuospatial sketch pad" (Baddeley, 1992). Baddeley (2012) proposed a revision of working memory model with four components by increasing the pleasure detector and Episodic buffer, the model is used to describe the influence of emotional factors on the working memory. The current working memory model and its basic theory research has got further development. People have a more in-depth understanding of the nature of working memory. But there are still some problems. For example, the model cannot explain how the storage and processing of sets of information are completed, the validation of Episodic buffer and the conception of Central Executive Function are not clear and so on. Therefore theoretical models of working memory still need to improve and supplement.

\subsection{Impairment of Working Memory in Patient with Depression}

There is evidence for the existence of cognitive impairment in patients with depression (Marazziti et al., 2010), while cognitive functions includes attention, working memory and executive function, Among them, working memory is an important component. The previous studies mainly focused on verbal and spatial working memory of patients with depression (Hasselbalch, Knorr, \& Kessing, 2011). But in recent years, studies on depressed patients with impairment of working memory turned to the core of working memory, namely the central executive, including inhibition, updating and shifting, these three parts are closely linked.

The core of effective functioning of the working memory is inhibition, and the main role of inhibition is to limit the relevant information into the working memory, delete irrelevant information and suppress erroneous information extracted from the working memory. Studies have shown that people with depression have cognitive inhibition impairment, especially the inhibition impairment about negative information materials (Joormann \& Gotlib, 2010). Joormann and Gotlib (2008) used the modified Sternberg working memory task to explore the updating of the contents of working memory and constrained capacity of working memory to exclude the irrelevant information, they found that when presented negative words, patients with depression showed stronger into effect than the control group. Gohier et al. (2009) found that impaired cognitive inhibition is associated with the severity of the depression of the patients with depression significantly. The patients with depression can't restrain neutral information into the working memory; their ability to limit and remove irrelevant working memory information is poor. And they proposed that the impairment of inhibition in patients may be the foundation of slower thought and attention deficit of the patients. Årdal and Hammar did a follow-up study up to 10 years, they found that the acute cognitive inhibition impairment of patients with depression is persistent, and this inhibition impairment may be a irreversible susceptibility mark of the recurrence of depression (Årdal \& Hammar, 2011). Therefore, the treatment of acute depression restrain impairment is critical, it's also the key to prevent the patient from further impairment to working memory.

The refreshing function of working memory affects the regulation of mood and assessment of personal experiences of the past (Pe, Raes, \& Kuppens, 2013). In order to test the ability of working memory to refresh emotional material, Levens and Gotlib (2010) asked subjects to complete 2-back task of the emotional faces, and also conducted a 0-back task as a perceptual process control. The results showed that compared with normal group, people with depression got out of grief stimulus more slowly and got out of a pleasant stimulus faster. Therefore, they think the difference of reaction time between groups may reflect the bias of the protection and Inadaptability of working memory and it influence the ability to regulate negative emotions. Lissnyder et al. (2012) studied the ability of working memory to transfer between internal emotional information and unemotional information. The results showed that depressive symptoms usually had nothing to do with impaired conversion ability. Meiran et al. (2011) studied the functions of patients with depression to inhibit, transfer and refresh. These functions have shown the impairment and indicated that the central executive of working memory was a whole, with consistency. 


\section{Functional Neuroimaging of Working Memory in Patients with Depression}

There is a impairment of working memory of depression, but the pathological basis of the working memory impairment in patients with depression is not yet clear. In recent years, with the development of functional brain imaging, especially the development of functional Magnetic Resonance Imaging (fMRI) and functional near infrared spectroscopy (fNIRS) provide conditions for us to understand its pathological mechanism. Impairment of working memory in patients with depression can be manifested in these functional brain imaging.

Numerous studies show that brain regions activated by working memory mainly located in Prefrontal cortex (PFC), parietal, supplementary motor area, Anterior cingulate cortex (ACC) and the reticular activating system.

Among them, the role of prefrontal cortex play in working memory is quite complex, including the functions of attention, inhibition, management and integration of memory information (Lisa \& Ebmeier, 2009). The dysfunction of dorsolateral prefrontal cortex, striatum and hippocampus would lead to the cognitive impairment such as attention, memory, working memory and executive function. DLPFC plays an important role in working memory and executive function. When patients with depression implement working memory task, it involves abnormal brain activation of the frontal edge of the network like DLPFC and ACC (Diener et al., 2012).

Researchers used neutral or emotional stimuli to explore the cognitive inhibition ability in patients with depression. they found that the counter-group difference was observed only in emotionally negative material presenting unpleasant scenes., and the damage of inhibition mechanism were followed by the changes of the functions of prefrontal dorsolateral prefrontal cortex and cingulate gyrus (Eugene et al., 2010). The study of Berman et al. (2011) found that the left inferior frontal gyrus was a key area to inhibit irrelevant information, while patients with depression in this region showed more activation of variation, which may lead to the lack of ability in patients with depression to suppress negative information.

Koster et al. (2011) proposed the hypothesis of mechanism from impairment, the key point of the hypothesis was that the time extension of self-related material processing was due to the attention cannot be divorced from the negative self-related information,

Foland-Ross et al. (2013) studied the neural basis of this mechanism from impairment and found that when patients with depression removed the negative words from working memory, the dorsal anterior cingulate, parietal cortex and bilateral insular cortices were significantly active. They think that the abnormal activation of these brain areas leads to the difficulty for patients with depression to remove negative stimuli from the working memory, which takes up more working memory resource.

Garrett et al. (2011) did fMRI scanning on patients with psychotic symptoms of depression and patients without psychotic symptoms of depression under the working memory task. The study found group without psychotic symptoms in depression showed abnormal in frontal executive area, the group with psychotic symptoms of depression showed abnormal activation in parahippocampal gyrus and the temporal-parietal region at lower demand levels, and temporal-parietal region is related to positioning unexpected stimuli. Therefore, they think this may reflect a nerve compensation for dysfunctional sensory gating.

Kerestes et al. (2012) did a fMRI study of remitted depressed patients, the study showed that during high working memory load, when response to negative emotional distracters, patients exhibited significantly greater activity relative to HC in the left DLPFC [Brodmann area (BA) 9/46]. By contrast, remitted depressed patients exhibited significantly lower activity in the right DLPFC and left VLPFC compared to HC when responsed to positive emotional distracters. This suggests that remitted depressed patients may continue to exhibit attentional biases.

Norbury \& Godlewska (2014) found that there was no significant difference in the working memory performance accuracy and response latency in remitted patients with depression. But bilateral hippocampus showed positive quadratic load and perturbed pattern of activation, which indicated a reduced ability to dampen task irrelevant activity might be the neurobiological factor of recurrent depression.

fNIRS is a relatively new brain imaging technique, which uses light-scattering properties of the main component of blood to 600 - $900 \mathrm{~nm}$ good near-infrared, resulting in the changes of oxygen hemoglobin and deoxygenated hemoglobin during the brain activity .

Schecklmanna et al. (2011) studied the unipolar and bipolar patients with depression in a working memory task with fNIRS over the prefrontal cortex. The results show that both patient groups showed diminished brain activity in all working memory conditions compared to healthy control. This suggested that depression would cause the function deficits of the brain, but the specific deficit was not known yet. So working memory processes or components can't differentiate unipolar from bipolar depression. 
The study of Pu et al. (2011) showed that patients showed a smaller increase in lateral prefrontal and superior temporal cortex activation during the working memory task and associated poorer task performance than healthy controls. The results contradicted with some previous fMRI studies. Moreover, they measured hemoglobin concentration changes of late-onset depression patients in the prefrontal and temporal regions during a working memory task using 52-channel near-infrared spectroscopy (NIRS). Patients were associated with reduced increase in prefrontal and temporal activation compared with healthy controls. And reduced activation in the prefrontal and temporal regions was significantly related to lower scores on the Social Adaptation Self-Evaluation Scale (SASS) in the patient group. These findings suggest that hemodynamic response in prefrontal and temporal regions during a working memory task may act as a biological marker of social functioning in late-onset patients (Pu et al., 2012).

\section{Future Research Orientations}

At present, research on working memory in patients with depression achieved some results at home and abroad, there is impairment of working memory in patients with depression, including various components of working memory, fMRI, fNIRS and other functional brain imaging techniques also confirmed the dysfunctions of related brain regions.

However, there are still many limitations in previous researches, and research about impairment of working memory in patients with depression is not very comprehensive, and it is generally limited to some portion of working memory component, such as the working memory capacity, spatial working memory or a subfunction of the central executive system. At present, there is no comprehensive review about research of all components of working memory in patients with depression at home and abroad. Meanwhile, the studies about working memory in different severity in patients with depression are also relatively small.

Although there were many Brain imaging studies about working memory on patients with depression, the brain mechanisms of working memory in patients with depression remains unclear, exactly which brain regions influence working memory capacity, the different impairment of working memory between subtypes of depression. the working memory damage is a cause or a result of depression, how to improve working memory by training patients with depression, and as the improvement of working memory, whether mood of the patients can turn good, these questions need to be explored further.

Thus, in future studies, we should do more to combine functional brain imaging techniques to explore the impairment mechanism of working memory in patients with depression, as well as the regulation of working memory to depression, and to figure out the causation between working memory and depression. We hope to improve the patient's mood by the improved training of working memory and analyze different subtypes' characteristics of impairment of working memory with the exclusion of patients' disease duration, age, sex, personality, habits, experience and other disturbing factors.

A variety of the effectively functional neuroimaging detection techniques, neuropsychological tests and comprehensive, scientific ways of studying in working memory model of depression provide the theoretical and scientific basis for the cure of depression.

\section{References}

Årdal, G., \& Hammar, Å. (2011). Is Impairment in Cognitive Inhibition in the Acute Phase of Major Depression Irreversible? Results from a 10-Year Follow-Up Study. Psychology and Psychotherapy: Theory, Research and Practice, 84, 141-150.

Baddeley, A. (2012). Working Memory: Theories, Models and Controversies. Annual Review of Psychology, 63, 1-29. http://dx.doi.org/10.1146/annurev-psych-120710-100422

Baddeley, A. (1992). Working Memory. Science, 255, 556-559. http://dx.doi.org/10.1126/science.1736359

Berman, M. G., Nee, D. E., Casement, M., Kim, H. S., Deldin, P., Kross, E. et al. (2011). Neural and Behavioral Effects of Interference Resolution in Depression and Rumination. Cognitive, Affective, \& Behavioral Neuroscience, 11, 85-96. http://dx.doi.org/10.3758/s13415-010-0014-X

Diener, C., Kuehner, C., Brusniak, W., Ubl, B., Wessa, M., \& Flor, H. (2012). A Meta-Analysis of Neurofunctional Imaging Studies of Emotion and Cognition in Major Depression. NeuroImage, 61, 677-685.

http://dx.doi.org/10.1016/j.neuroimage.2012.04.005

Eugene, F., Joormann, J., Cooney, R. E., Atlas, L. Y., \& Gotlib, I. H. (2010). Neural Correlates of Inhibitory Deficits in Depression. Psychiatry Research: Neuroimaging, 181, 30-35. http://dx.doi.org/10.1016/j.pscychresns.2009.07.010 
Foland-Ross, L. C., Hamilton J. P., Joormann, J., Berman, M. G., Ian, J. J., \& Gotlib, H. (2013). The Neural Basis of Difficulties Disengaging From Negative Irrelevant Material in Major Depression. Psychological Science, 24, 334-344. http://dx.doi.org/10.1177/0956797612457380

Garrett, A., Kelly, R., Gomez, R., Keller, J., Schatzberg, A. F., \& Reiss, A. L. (2011). Aberrant Brain Activation during a Working Memory Task in Psychotic Major Depression. The American Journal of Psychiatry, 168, 173-182. http://dx.doi.org/10.1176/appi.ajp.2010.09121718

Gohier, B., Ferracci, L., Surguladze, S. A., Lawrence, E., El Hage, W., Kefi, M. Z. et al. (2009). Cognitive Inhibition and Working Memory in Unipolar Depression. Journal of Affective disorder, 116, 100-105. http://dx.doi.org/10.1016/j.jad.2008.10.028

Hasselbalch, B. J., Knorr, U., \& Kessing, L.V. (2011). Cognitive Impairment in the Remitted State of Unipolar Depressive Disorder: A Systematic Review. Journal of Affective Disorders, 134, 20-31. http://dx.doi.org/10.1016/j.jad.2010.11.011

Joormann, J., \& Gotlib, I. H. (2008). Updating the Content of Working Memory in Depression: Interference from Irrelevant Negative Material. Journal of Abnormal Psychology, 117, 182-192. http://dx.doi.org/10.1037/0021-843X.117.1.182

Joormann, J., \& Gotlib, I. H. (2010). Emotion Regulation in Depression: Relation to Cognitive Inhibition. Cognition and Emotion, 24, 281-298. http://dx.doi.org/10.1080/02699930903407948

Kerestes, R., Ladouceur, C. D., Meda, S., Nathan, P. J., Blumberg, H. P., Maloney, K. et al. (2012). Abnormal Prefrontal Activity Subserving Attentional Control of Emotion in Remitted Depressed Patients during a Working Memory Task with Emotional Distracters. Psychological Medicine, 42, 29-40. http://dx.doi.org/10.1017/S0033291711001097

Koster, E. H. W., Lissnyder, E. D., Derakshan, N., \& De Raedt, R. (2011). Understanding Depressive Rumination from a Cognitive Science Perspective: The Impaired Disengagement Hypothesis. Clinical Psychology Review, 31, 138-145. http://dx.doi.org/10.1016/j.cpr.2010.08.005

Levens, S., \& Gotlib, I. (2010). Updating Positive and Negative Stimuli in Working Memory in Depression. Journal of Experimental Psychology: General, 39, 654-664. http://dx.doi.org/10.1037/a0020283

Lisa, M. M., \& Ebmeier, K. P. (2009). A Meta-Analysis of Depression Severity and Cognitive Function. Journal of Affective Disorders, 119, 1-8.

Lissnyder, E. D., Koster, E. H. W., \& Raedt, R. D. (2012). Emotional Interference in Working Memory Is Related to Rumination. Cognitive Therapy and Research, 36, 348-357. http://dx.doi.org/10.1007/s10608-011-9352-4

Marazziti, D., Consoli, G., Picchetti, M., Carlini, M., \& Faravelli, L. (2010). Cognitive Impairment in Major Depression. European Journal of Pharmacology, 629, 83-86. http://dx.doi.org/10.1016/j.ejphar.2009.08.046

Meiran, N., Diamond, G. M., Toder, D., \& Nemets, B. (2011). Cognitive Rigidity in Unipolar Depression and Obsessive Compulsive Disorder: Examination of Task Switching, Stroop, Working Memory Updating and Post-Conflict Adaptation. Psychiatry Research, 185, 149-156. http://dx.doi.org/10.1016/j.psychres.2010.04.044

Norbury, R., Godlewska, B., \& Cowen, P. J. (2014). When Less Is More: A Functional Magnetic Resonance Imaging Study of Verbal Working Memory in Remitted Depressed Patients. Psychological Medicine, 44, 1197-1203. http://dx.doi.org/10.1017/S0033291713001682

Pe, M. L., Raes, F., \& Kuppens, P. (2013). The Cognitive Building Blocks of Emotion Regulation: Ability to Update Working Memory Moderates the Efficacy of Rumination and Reappraisal on Emotion. PloS ONE, 8, e69071. http://dx.doi.org/10.1371/journal.pone.0069071

Pu, S., Yamada, T., Yokoyama, K., Matsumura, H., Kobayashi, H., Sasaki, N. et al. (2011). A Multi-Channel Near-Infrared Spectroscopy Study of Prefrontal Cortex Activation during Working Memory Task in Major Depressive Disorder. Neuroscience Research, 70, 91-97. http://dx.doi.org/10.1016/j.neures.2011.01.001

Pu, S., Yamada, T., Yokoyama, K., Matsumura, H., Mitani, H., Adachi, A. et al. (2012). Reduced Prefrontal Cortex Activation during the Working Memory Task Associated with Poor Social Functioning in Late-Onset Depression: Multi-Channel Near-Infrared Spectroscopy Study. Psychiatry Research: Neuroimaging, 203, 222-228. http://dx.doi.org/10.1016/j.pscychresns.2012.01.007

Schecklmanna, M., Dreslerb, T., Beck, S., Jay, J. T., Febres, R., Haeusler, J. et al. (2011). Reduced Prefrontal Oxygenation during Object and Spatial Visual Working Memory in Unipolar and Bipolar Depression. Psychiatry Research: Neuroimaging, 194, 378-384. http://dx.doi.org/10.1016/j.pscychresns.2011.01.016 